\title{
Molecular characterization of Aspergillus flavus and aflatoxin contamination of wheat grains from Saudi Arabia
}

\author{
A.S. Al-Wadai ${ }^{1}$, M.R. Al-Othman ${ }^{1}$, M.A. Mahmoud ${ }^{1,2}$ and \\ A.R.M. Abd El-Aziz ${ }^{1}$ \\ ${ }^{1}$ Botany and Microbiology Department, College of Science, \\ King Saud University, Riyadh, Saudi Arabia \\ ${ }^{2}$ Plant Pathology Research Institute, Agricultural Research Center, Giza, Egypt \\ Corresponding author: M.A. Mahmoud \\ E-mail:momahmoud@ksu.edu.sa
}

Genet. Mol. Res. 12 (3): 3335-3352 (2013)

Received December 19, 2012

Accepted July 19, 2013

Published September 3, 2013

DOI http://dx.doi.org/10.4238/2013.September.3.10

\begin{abstract}
Twelve species belonging to six fungal genera were found to be associated with wheat (Triticum aestivum L.) grain samples collected from three main regions in Saudi Arabia. The most common genera (average frequency) were Aspergillus (14.3\%), Fusarium (29.1\%), Penicillium (9.3\%), and Alternaria (8.2\%). Nineteen isolates of Aspergillus flavus were screened for their ability to produce aflatoxins using HPLC. Thirteen isolates produced aflatoxins ranging from 0.5 to $2.6 \mu \mathrm{g} / \mathrm{kg}$. Inter-simple sequence repeats (ISSR), and random amplified polymorphic DNA (RAPD) molecular markers were used, with the aim of genetically characterizing strains of $A$. flavus to discriminate between aflatoxigenic and non-aflatoxigenic isolates. RAPD and ISSR analysis revealed a high level of genetic diversity in the A. flavus population, useful for genetic characterization. Clustering based on RAPD and ISSR dendograms was unrelated to geographic origin. RAPD and
\end{abstract}


ISSR markers were not suitable to discriminate aflatoxigenic and nonaflatoxigenic isolates, but ISSR primers were better compared to RAPD.

Key words: Aspergillus flavus; Random amplified polymorphic DNA; Inter-simple sequence repeats; Aflatoxin; Wheat; HPLC

\section{INTRODUCTION}

Wheat is one of the most important and strategic products in all the world, where it can provide more than $20 \%$ of the calories of daily food intake (Wiese, 1987). Wheat is grown in most parts of the world, from near-arctic to near-equatorial latitudes. It is the most important crop among the cereals by area planted and is followed in importance by corn, barley, and sorghum. The amount of wheat traded internationally exceeds that of all other grains. Furthermore, the protein and caloric content of wheat is greater than that of any other food crop. Most wheat is consumed in the form of baked goods, mainly bread; therefore, wheat grains must be milled to produce flour prior to consumption.

Wheat is also used as an ingredient in compound feedstuffs, starch production, and as a feed stock in ethanol production (FAO, 2009). Aflatoxins (AFs) are toxic secondary metabolites produced by Aspergillus flavus, $A$. parasiticus, $A$. nomius, $A$. pseudotamarii, A. bombycis, A. toxicarius, A. minisclerotigenes, A. parvisclerotigenus, and A. arachidicola in the Aspergillus section Flavi (Pildain et al., 2008). AFs can enter the food chain in the field, during storage, or later, under favorable conditions of temperature and humidity. The most potent of the four naturally occurring AFs (B1, B2, G1 and G2) is B1, which is listed as a group I carcinogen by the International Agency for Research on Cancer (IARC, 1982) because of its demonstrated carcinogenicity in humans (Castegnaro and Wild, 1995). AFs are carcinogens, mutagens, and teratogens (Kotsonis et al., 2001). Contamination of food and feed with AFs represents a high risk for human and animal health (Moss, 1996). Furthermore, mycotoxins are responsible for generating huge economic losses in the producing countries (Bhat and Vasanthi, 2003). Pittet (1998) reported that $25-40 \%$ of cereals consumed in the world are contaminated by these toxic compounds.

RAPD markers have found a wide range of applications in genetic diversity, characterization, genetic structure of populations, and genome mapping. This is mainly due to the speed, cost, and efficiency of the RAPD technique to generate large numbers of markers in a short period, compared with other methods (Bardakci, 2001). Besides, with RAPD, the appearance of new ligation sites only creates new annealing sites without impairing pre-existing ones, which would continue to be amplified. Consequently, RAPD allows an independence of characters, which is a clear advantage over techniques that directly or indirectly make use of restriction enzymes, such as restriction fragment length polymorphism. RAPD markers have detected genetic variability between the isolates of A. flavus and related species (Batista et al., 2008; Reddy et al., 2009; Irshad and Nawab, 2012). RAPD markers have been used to discriminate between aflatoxigenic from non-aflatoxigenic isolates of A. flavus (Lourenço et al., 2007; Gashgari et al., 2010). The inter-simple sequence repeat (ISSR) markers are highly polymorphic and are useful in studies on genetic diversity, phylogeny, gene tagging, genome mapping, and evolutionary biology (Reddy et al., 2002). The ISSR technique is a polymerase chain reaction (PCR)-based method that involves amplification of a DNA segment present at an amplifiable distance between two identical microsatellite repeat regions. Different isolates 
can be distinguished from each other on the basis of differences in repeat numbers. Microsatellite markers are easily amplified by PCR using primers based on their flanking sequences. The number of repeats in each marker can be deduced from the sizes of the fragments. All repeat numbers of the analyzed markers form a genotype for each individual isolate. These genotypes are easily compared to each other (Hadrich et al., 2011). ISSR markers are very useful for investigating the diversity and population structure of A. flavus and related species (Tran-Dinh and Carter, 2000; Batista et al., 2008; Hadrich et al., 2010; Neal et al., 2011). ISSR markers have been used to determine similarity and dissimilarity between aflatoxigenic and non-aflatoxigenic isolates of $A$. flavus (Hatti et al., 2010).

The aims of the present study were: 1) to genetically characterize 19 isolates of the $A$. flavus group isolated from the wheat crop found in three main regions in Saudi Arabia, 2) to determine the genetic relationship (comparing the genomic profile) between aflatoxigenic and nonaflatoxigenic isolates using PCR amplification of RAPD and ISSR molecular markers, and 3) to determine the AFs in these isolates using high-performance liquid chromatography (HPLC).

\section{MATERIAL AND METHODS}

\section{Collection of samples}

Fifteen samples ( $250 \mathrm{~g}$ each) of each grain type were collected from different markets located in Riyadh, Dammam, and Abha, in the Kingdom of Saudi Arabia, during 2012 for this experimental study. The samples were stored at $2^{\circ} \mathrm{C}$ until being used (Czerwiecki et al., 2002).

\section{Isolation, purification, and identification of pathogen}

Samples were surface sterilized with 5\% sodium hypochlorite solution for $1 \mathrm{~min}$, before they were rinsed three times with sterile distilled water. Five grains were placed randomly on potato dextrose agar (PDA) on three 9-cm Petri dishes. The Petri dishes were incubated at $25^{\circ} \mathrm{C}$ and observed daily for emergence of colonies for 5 days, after which the colonies were counted. Isolates were purified either by single spore or hyphal tip methods and then transferred to PDA slants. All the isolates of fungal species were identified up to the species level using keys and manuals (Raper and Fennell, 1965; Domsch et al., 1993). The isolation frequencies of fungal species were calculated according to the method of Gonzalez et al. (1995). Identification of fungal isolates was carried out on the basis of morphological and microscopic characteristics at the Regional Center for Mycology and Biotechnology, Al-Azhar University, Cairo, Egypt.

\section{Aflatoxin production in different culture media and detection with fluorescence}

For determination of AF production based on medium fluorescence, all Aspergillus strains were cultivated on PDA at $25^{\circ} \mathrm{C}$ for 7 days. A mycelial plug of each strain was placed at the center of a Petri dish containing test medium [PDA, Czapek agar (CZ), and malt extract agar (Davis et al., 1987). The plates were incubated at $25^{\circ} \mathrm{C}$ for 4 days in the dark, and the presence or absence of fluorescence on the agar surrounding the growing Aspergillus colonies was determined by exposing the Petri dishes to ultraviolet (UV) light $(365 \mathrm{~nm})$ and expressed as positive or negative according to Franco et al. (1998). 


\section{Determination of aflatoxins by HPLC}

Isolates were grown in sterile SMKY liquid medium (20 g sucrose, $0.5 \mathrm{~g}$ magnesium sulfate, $3 \mathrm{~g}$ potassium nitrate, $7 \mathrm{~g}$ yeast extract, and $1000 \mathrm{~mL}$ distilled water) (Davis et al., 1987). The flasks were inoculated with 6-mm diameter discs of toxigenic Aspergillus spp at $25^{\circ}$ $\pm 2^{\circ} \mathrm{C}$ for 7 days (Paranagama et al., 2003), with three replicates. After incubation, the content of each flask was filtered (Whatman No. 1). For AF extraction, the filtrate of each flask was treated three times with $50 \mathrm{~mL}$ chloroform in a separatory funnel. The chloroform extract was separated, dehydrated with anhydrous sodium sulfate and evaporated to dryness on a water bath at $50^{\circ} \mathrm{C}$ under vacuum. The residues were dissolved in $10 \mathrm{~mL}$ methanol and stored in dark vials.

The extract was passed through a $0.45-\mu \mathrm{m}$ micro-filter. Analysis of compounds was performed by HPLC (Perkin Elmer model series 200 UV/VIS) with a C18 column (300 mm x $3.9 \mathrm{~mm}, 4 \mu \mathrm{m}$ ). The HPLC system was equipped with a UV detector and fluorescence with 365 $\mathrm{nm}$ excitation and 430 emission wavelengths. The mobile phase consisted of methanol:acetic acid:water $(20: 20: 60, \mathrm{v} / \mathrm{v} / \mathrm{v})$. The total run time for the separation was approximately $25 \mathrm{~min}$ at a flow rate of $1 \mathrm{~mL} / \mathrm{min}(\mathrm{Gertz}, 1990)$.

\section{Extraction of DNA from $A$. flavus isolates}

A. flavus isolates were cultured on double-layer media on 50-mm Petri dishes, one solid and the other liquid. The solid base medium was PDA as a film, and the top medium, liquid, was $1200 \mu \mathrm{L}$ peptone yeast glucose. Fungi were incubated at $25^{\circ} \mathrm{C}$ for two days. Fungal mycelia (50 $\mathrm{mg}$ ) were scraped using slide cover slips and transferred to $1.5-\mathrm{mL}$ sterile Eppendorf tubes for DNA isolation. DNA was extracted from $50 \mathrm{mg}$ fresh mat according to Amer et al. (2011).

\section{RAPD by PCR}

To identify the best primers to establish the RAPD profile, we tested 6 primers from the standard RAPD primer kits (Amersham Pharmacia), using total DNA from A. flavus isolates as template. The three primers selected with high reproducibility and clear banding profiles were the RAPD primers 1,3 , and 5 . The PCR mixtures were made to a final volume of $25 \mu \mathrm{L}$, containing reaction buffer ( $20 \mathrm{mM}$ Tris- $\mathrm{HCl}$, pH 8.4, $50 \mathrm{mM} \mathrm{KCl}$ ), $3.4 \mathrm{mM} \mathrm{MgCl}, 0.25 \mathrm{mM}$ dNTP, 0.4 $\mathrm{mM}$ of each primer, $2 \mathrm{U} \mathrm{Taq}$ DNA polymerase (BioLabs), and $25 \mathrm{ng}$ genomic DNA (Williams et al., 1990). Amplification consisted of an initial denaturation step at $95^{\circ} \mathrm{C}$ for $5 \mathrm{~min}$ (one cycle) followed by 45 cycles of denaturation at $95^{\circ} \mathrm{C}$ for $1 \mathrm{~min}$, annealing at $36^{\circ} \mathrm{C}$ for $1 \mathrm{~min}$, and amplification at $72^{\circ} \mathrm{C}$ for $2 \mathrm{~min}$, with a final extension at $72^{\circ} \mathrm{C}$ for $5 \mathrm{~min}$ (one cycle). PCR products were separated by $1.5 \%$ agarose gel electrophoresis in $1 \mathrm{X}$ TAE buffer (Tris-acetate EDTA, pH 8.0 ) at $100 \mathrm{~V}$ for $50 \mathrm{~min}$, using a 500-bp ladder DNA marker (Intron, Korea). DNA was visualized by UV light after staining with ethidium bromide. The primers tested are shown in Table 1.

\section{ISSR by PCR}

PCR amplification of ISSR was performed with (GTG) $)_{5},(\mathrm{GACA})_{4}$, and (AGAG) $)_{4} \mathrm{G}$ primers. The reaction mixtures were made to a final volume of $25 \mu \mathrm{L}$, containing reaction buffer (20 mM Tris-HCl, pH 8.4, $50 \mathrm{mM} \mathrm{KCl}$ ), $1.5 \mathrm{mM} \mathrm{MgCl}, 0.25 \mathrm{mM} \mathrm{dNTP}, 0.25 \mathrm{mM}$ primer, 
1.25 U Taq DNA polymerase (BioLabs), and $25 \mathrm{ng}$ genomic DNA (Williams et al., 1990). Amplification consisted of an initial denaturation step at $93^{\circ} \mathrm{C}$ for $5 \mathrm{~min}$, followed by 40 cycles of denaturation at $93^{\circ} \mathrm{C}$ for $20 \mathrm{~s}$, annealing at $55^{\circ} \mathrm{C}$ for $45 \mathrm{~s}$, and amplification at $72^{\circ} \mathrm{C}$ for 90 $\mathrm{s}$, with a final extension at $72^{\circ} \mathrm{C}$ for 6 min. The primers tested are shown in Table 1.

Table 1. Primers used to amplify Aspergillus flavus DNA.

\begin{tabular}{llll}
\hline Primer code & \multicolumn{2}{c}{ RAPD primers } \\
\cline { 2 - 4 } & Sequence & Amplified region & References \\
\hline RAPD primer 1 & 5'-GGT GCG GGA A-3' & Fragments from 1-10 genomic sites simultaneously & Mahmoud et al., 2012 \\
RAPD primer 2 & 5'-GTT TCG CTC C-3' & & \\
RAPD primer 5 & 5'-AAC GCG CAA C-3' & \multicolumn{1}{c}{ ISSR primers } & References \\
\hline Primer code & & Amplified region & Batista et al., 2008 \\
\cline { 2 - 4 } & Sequence & Minisatellite-region DNA \\
\hline (GTG $)_{5}$ & 5'-GTG GTG GTG GTG GTG-3' & & \\
$(\text { GACA })_{4}$ & 5'-GAC AGA CAG ACA GAC A-3' & & \\
$\left(\right.$ AGAG) ${ }_{4} G$ & 5'-AGA GAG AGA GAG AGA GG-3' & & \\
\hline
\end{tabular}

\section{DNA electrophoresis}

For all samples, the amplified DNA $(15 \mu \mathrm{L})$ was electrophoresed (Wide Mini-SubCell GT System, Bio-RAD) on $2 \%$ agarose gels containing $0.5 \mu \mathrm{g} / \mathrm{mL}$ ethidium bromide, at a constant $75 \mathrm{~V}$ and $60 \mathrm{~mA}$, and visualized with UV transilluminator.

\section{Gel analysis for DNA}

The DNA gel was scanned for band Rf using a gel documentation system (AAB Advanced American Biotechnology, Fullerton, CA, USA). The different molecular weights of the bands were determined against a DNA standard (kb DNA ladder, Stratagene, Canada) with molecular weights 100, 200, 300, 400, 500, 600, 700, 800, 900, 1000, and $1500 \mathrm{bp}$. The similarity level was determined by unweighted pair group method with arithmetic mean (UPGMA).

\section{RESULTS}

\section{Frequency percentage of isolated fungi in wheat grains from Riyadh, Dammam, and Abha regions in Saudi Arabia}

Twelve species belonging to six fungal genera were obtained from the test samples (Table 2). In the Riyadh region, A. flavus, Alternaria longipes, and Fusarium moniliforme were the dominant fungi isolated from wheat grains, with a frequency percentage of $33.4,12.5$, and $12.5 \%$, respectively. The other fungi isolated occurred in frequencies ranging from 0.00 to $8.30 \%$. In the Dammam region, A. flavus, F. moniliforme and Fusarium solani were the dominant fungi isolated from wheat grains, with a frequency percentage of $30.40,13.10$, and $8.70 \%$, respectively. The other fungi isolated occurred in a frequency percentage ranging from 0.00 to $8.70 \%$. In Abha region, A. flavus, F. moniliforme, and Penicillium implicatum were the dominant fungi isolated from wheat grains, with a frequency percentage of $30.70,15.40$ and $15.40 \%$, respectively. 


\begin{tabular}{|c|c|c|c|}
\hline Isolated fungi & Riyadh & Dammam & Abha \\
\hline Aspergillus flavus & 33.40 & 30.40 & 30.70 \\
\hline A. niger & 4.20 & 8.70 & 7.70 \\
\hline A. parasiticus & 0.00 & 8.70 & 0.00 \\
\hline Alternaria longipes & 12.50 & 0.00 & 7.70 \\
\hline A. chlamydospora & 0.00 & 4.30 & 0.00 \\
\hline Fusarium moniliforme & 12.50 & 13.10 & 15.40 \\
\hline F. oxysporum & 8.30 & 13.10 & 0.00 \\
\hline F. solani & 8.30 & 8.70 & 7.70 \\
\hline Penicillium funiculosum & 0.00 & 4.30 & 0.00 \\
\hline P. implicatum & 8.30 & 0.00 & 15.40 \\
\hline Eupenicillium alutaceum & 4.20 & 8.70 & 7.70 \\
\hline Rhizopus stolonifer & 8.30 & 0.00 & 7.70 \\
\hline Total & 100.00 & 100.00 & 100.00 \\
\hline
\end{tabular}

The other fungi isolated occurred in a frequency percentage ranging from 0.00 to $7.70 \%$. A. flavus isolates were the dominant fungi in these three regions. A. flavus isolates included 8 isolates from Riyadh, 7 from Dammam and 4 from Abha.

\section{Detection of aflatoxigenic $A$. flavus isolates under UV irradiation (365 nm)}

Three culture media, namely CZ, PDA, and yeast extract sucrose agar (YES), were used to screen for AF production. Production of AFs was readily detectable by direct visualization of a beige ring surrounding colonies after 4 days of incubation. The presence or absence of fluorescence on the agar surrounding the colonies assayed was determined under UV irradiation $(365 \mathrm{~nm})$ and expressed as positive or negative. Data in Table 3 show that 15 isolates of A. flavus tested positive for AF production while 4 were negative.

Table 3. Detection of aflatoxigenic Aspergillus flavus isolates under UV radiation (365 nm).

\begin{tabular}{|c|c|c|c|}
\hline A. flavus code & $\mathrm{PDA}+\mathrm{NaCl}$ & $\mathrm{CZ}$ & YES \\
\hline \multicolumn{4}{|l|}{ Riyadh region } \\
\hline W1 & - & + & + \\
\hline W2 & - & + & + \\
\hline W3 & - & - & + \\
\hline W4 & - & - & - \\
\hline W5 & - & + & + \\
\hline W6 & - & + & - \\
\hline W7 & - & - & - \\
\hline W8 & + & - & + \\
\hline \multicolumn{4}{|c|}{ Dammam region } \\
\hline W9 & - & - & + \\
\hline W10 & - & - & + \\
\hline W11 & - & - & - \\
\hline W12 & - & - & + \\
\hline W13 & - & - & + \\
\hline W14 & - & + & + \\
\hline W15 & - & - & + \\
\hline \multicolumn{4}{|l|}{ Abha region } \\
\hline W16 & - & + & - \\
\hline W17 & - & - & - \\
\hline W18 & - & - & + \\
\hline W19 & - & - & + \\
\hline
\end{tabular}

$\mathrm{PDA}=$ potato dextrose agar; $\mathrm{CZ}=$ Czapek Dox agar; $\mathrm{YES}=$ yeast extract sucrose agar. 


\section{Aflatoxin production}

Nineteen isolates were capable of producing detectable levels of both B and G AFs (AFB and AFG), although six isolates failed to produce any detectable amount. The highest level (2.3 ppb) of B1 was obtained from isolate W10 while the highest level (2.6 ppb) of B2 was obtained from isolates $\mathrm{W} 10$ and $\mathrm{W} 15$. Isolate $\mathrm{W} 2$ was the highest producer (1.3 ppb) of G1 while isolate $\mathrm{W} 1$ was the highest producer $(0.5 \mathrm{ppb})$ of G2 (Table 4).

\begin{tabular}{|c|c|c|c|c|}
\hline \multirow[t]{2}{*}{ A. flavus code* } & \multicolumn{4}{|c|}{ Aflatoxin (ppb) } \\
\hline & B1 & B2 & G1 & G2 \\
\hline \multicolumn{5}{|l|}{ Riyadh region } \\
\hline W1 & 1.9 & 2.1 & 1.1 & 0.5 \\
\hline W2 & 1.7 & 2.1 & 1.3 & 0.0 \\
\hline W3 & 1.8 & 2.0 & 0.9 & 0.3 \\
\hline W5 & 2.0 & 1.6 & 0.9 & 0.4 \\
\hline W6 & 1.8 & 1.5 & 0.0 & 0.0 \\
\hline W8 & 1.6 & 1.8 & 0.8 & 0.3 \\
\hline \multicolumn{5}{|l|}{ Dammam region } \\
\hline W9 & 0.0 & 0.0 & 1.2 & 0.2 \\
\hline W10 & 2.3 & 2.6 & 0.9 & 0.3 \\
\hline W12 & 1.7 & 0.0 & 0.0 & 0.0 \\
\hline W13 & 0.0 & 0.0 & 0.0 & 0.0 \\
\hline W14 & 1.8 & 2.1 & 1.0 & 0.3 \\
\hline \multicolumn{5}{|l|}{ Abha region } \\
\hline W15 & 0.0 & 2.6 & 0.9 & 0.1 \\
\hline W16 & 1.2 & 0.0 & 0.7 & 0.0 \\
\hline W18 & 1.1 & 0.7 & 0.0 & 0.2 \\
\hline W19 & 0.0 & 0.0 & 0.0 & 0.0 \\
\hline
\end{tabular}

*W4, W7, W11, W17 tested negative for aflatoxin and were not cited in Table.

\section{Chemotype patterns}

A. flavus isolates were classified into eight chemotypes (I to VIII) on the basis of their ability to produce B1, B2, G1, and G2 (Table 5). Chemotype I (aflatoxigenic isolates) was the first prominent group comprising $36.8 \%$ of the isolates. Only seven isolates produced all AFs (chemotype I). Chemotype VIII (non-aflatoxigenic) was the second prominent group comprising $31.6 \%$ of the isolates. The percentage of each remaining chemotype was $5.26 \%$. One isolate produced B1 and B2 (chemotype II) and one isolate also produced both G1 and G2 (chemotype III). B1 was produced by one isolate (chemotype IV) and B1 and G1 were produced by another isolate (chemotype VI). One isolate produced B2, G1, and G2 (chemotype $\mathrm{V}$ ) and another isolate produced B1, B2, and G2 (chemotype VII).

\section{Genetic characterization of $A$. flavus isolates}

Genetic characterization was evaluated by PCR amplification using a set of 6 primers (3 RAPD and 3 ISSR). The amplification products were analyzed for polymorphisms by gel electrophoresis to determine whether pathotypes could be distinguished at the molecular level. 
Table 5. Chemotype patterns of Aspergillus flavus isolated from Riyadh, Dammam, and Abha regions based on the ability for producing aflatoxins B1, B2, G1, and G2.

\begin{tabular}{lccccc}
\hline Chemotype & \multicolumn{3}{c}{ Aflatoxin } & No. (\%) of isolates \\
\cline { 2 - 5 } & B1 & B2 & G1 & G2 & \\
\hline I & + & + & + & + & $7(36.8)$ \\
II & + & + & - & - & $1(5.26)$ \\
III & - & - & + & + & $1(5.26)$ \\
IV & + & + & - & - & $1(5.26)$ \\
V & + & - & + & - & $1(5.26)$ \\
VI & + & + & - & + & $1(5.26)$ \\
VII & - & - & - & - & $1(5.26)$ \\
VIII & & & & & $6(31.6)$ \\
\hline
\end{tabular}

\section{Phenetic analysis by three RAPD primers}

\section{Phenetic analysis of 19 A. flavus isolates by RAPD primer 1}

Dendrogram analysis grouped the isolates into two main clusters with genetic similarity (GS) of $70.70 \%$ based on the banding pattern (Figure 1). GS between the isolates tested ranged from 70.70 to $99.85 \%$ (Figure 1). The first main cluster included the two isolates W2 and W4 from the Riyadh region, which showed a high GS of $98.71 \%$. The second main cluster included two groups at $76.21 \%$ GS. The first one included Abha isolates W16, W18, and W19 with $97.11 \%$ GS. The second group included the remaining isolates with GS ranging from 86.27 to $99.77 \%$. The second group was divided into two subgroups at $86.27 \%$. The first subgroup included the Dammam isolates W11, W12, and W15. The second subgroup was also divided into two subclusters. The first one included only isolate W17 from Abha. The second subcluster branched into overlapping subclusters with GS ranging from 92.37 to $99.77 \%$. In these subclusters, the isolates that represented the Riyadh region, i.e., isolates $\mathrm{W} 8$ and $\mathrm{W} 6$, showed more than $95 \%$ GS between each other and isolates W1 and W7 displayed more than 99\% GS between each other. The isolates representing the Dammam region, i.e., isolates W10 and W13, showed more than $99 \%$ GS between each other. This fact indicated that this primer was somewhat able to differentiate those regions. With reference to this primer, there was partial relationship between clustering in the RAPD dendrogram and geographic origin of the isolates tested.

Six subclusters included 12 isolates, 9 isolates producing AFB and AFG and 3 isolates not producing AFB and AFG, isolated from the Riyadh, Dammam, and Abha regions, and they resided in two main clusters with a random distribution. Five subclusters included a combination of ten aflatoxigenic and non-aflatoxigenic isolates, for example, one subcluster included the two isolates W1 (producing AFB and AFG) and W7 (non-producing AFB and AFG), which exhibited a 99.77\% GS. Only one subcluster included the two aflatoxigenic isolates W16 and W18, which showed a $99.85 \%$ GS.

AFB- and AFG-producing isolates resided in both clusters with a random distribution and there was no obvious correlation between toxigenicity and cluster patterns of the isolates. On the basis of the dendrogram data, an exact association was not established between RAPD genotypes and the ability to produce AFB and AFG. 


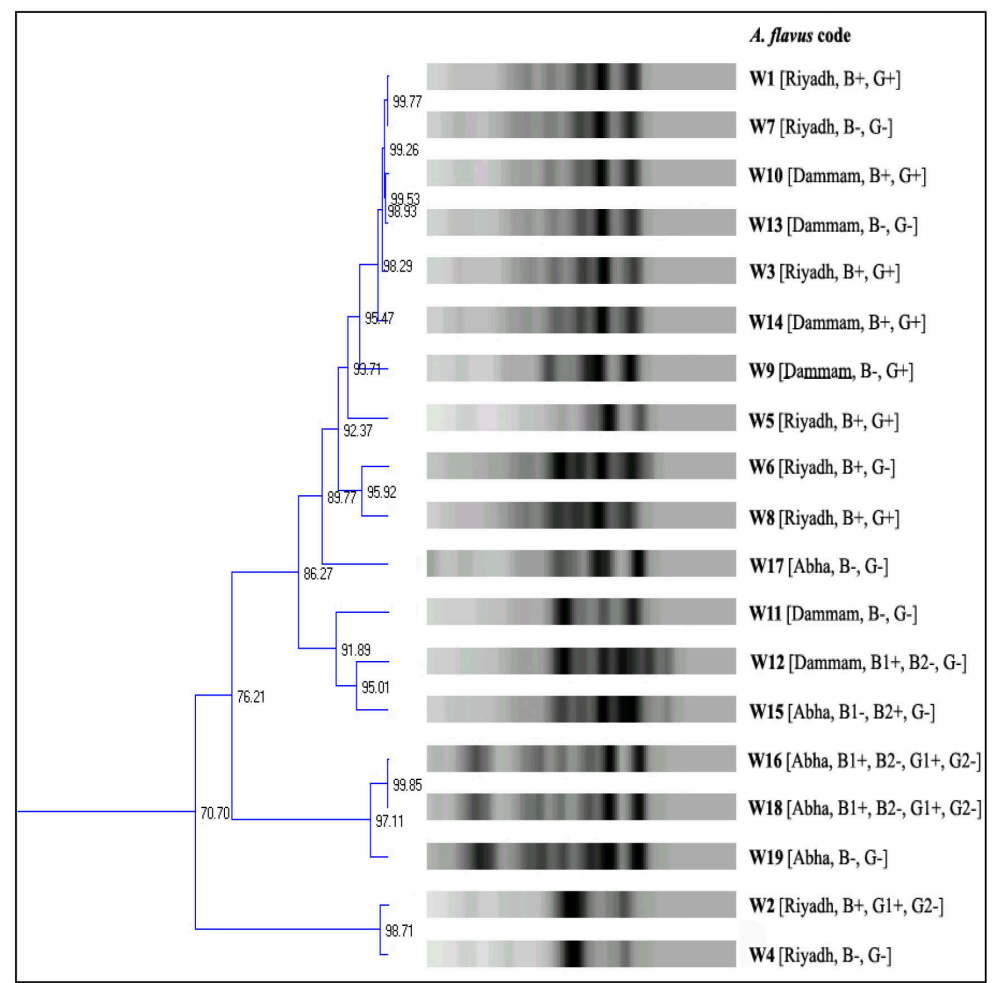

Figure 1. Dendrogram obtained by UPGMA method derived from PCR amplification banding of RAPD with RAPD primer 1 of 19 Aspergillus flavus isolates.

\section{Phenetic analysis of 19 A. flavus isolates using RAPD primer 3}

Using RAPD primer 3, cluster analysis placed the 19 A. flavus isolates into two main clusters with similarity of 58.70-99.22\% (Figure 2). The first main cluster included two groups at $77.68 \%$ GS, which included two subclusters. The first subcluster included Riyadh isolates 1 , 5,2 , and 3 with GS ranging from 86.12 to $95.69 \%$. The second subcluster included Abha isolates $16,17,18,19$, and Riyadh isolate 4, with GS ranging from 89.94 to $98.76 \%$. The second main cluster included two groups at $73.84 \% \mathrm{GS}$; the first one included isolates 7 and 9 with $98.60 \% \mathrm{GS}$, and the second group included two subclusters at $82.42 \%$ GS. The first subclusters included Riyadh region isolates 6 and 8 and Dammam region isolates 10,14, and 15, with GS ranging from 94.01 to $99.22 \%$. The second subclusters included Abha region isolates 11, 12 , and 13 , with GS ranging from 92.49 to $96.69 \%$.

Remarkably, this primer succeeded in grouping the Riyadh and Abha isolates in the same group but, not the Dammam isolates. This indicated that this primer was able to differentiate their regions somewhat. With reference to this primer, there was partial relationship between clustering in the RAPD dendrogram and geographic origin of the isolates tested.

Five subclusters included 10 isolates, 6 isolates producing AFB and AFG and 4 isolates not producing AFB and AFG, obtained from the Riyadh, Dammam, and Abha regions, and they 
resided in two main clusters with a random distribution. Three subclusters included six isolates in a combination of aflatoxigenic and non-aflatoxigenic; for example, one subcluster included the two isolates W17 (AFB- and AFG-non-producing) and W18 (AFB- and AFG-producing), which showed $98.756 \% \mathrm{GS}$, and two subclusters included four aflatoxigenic isolates, including W1 and W5 with $95.69 \%$ GS. There was no correlation between DNA banding pattern and AF production.

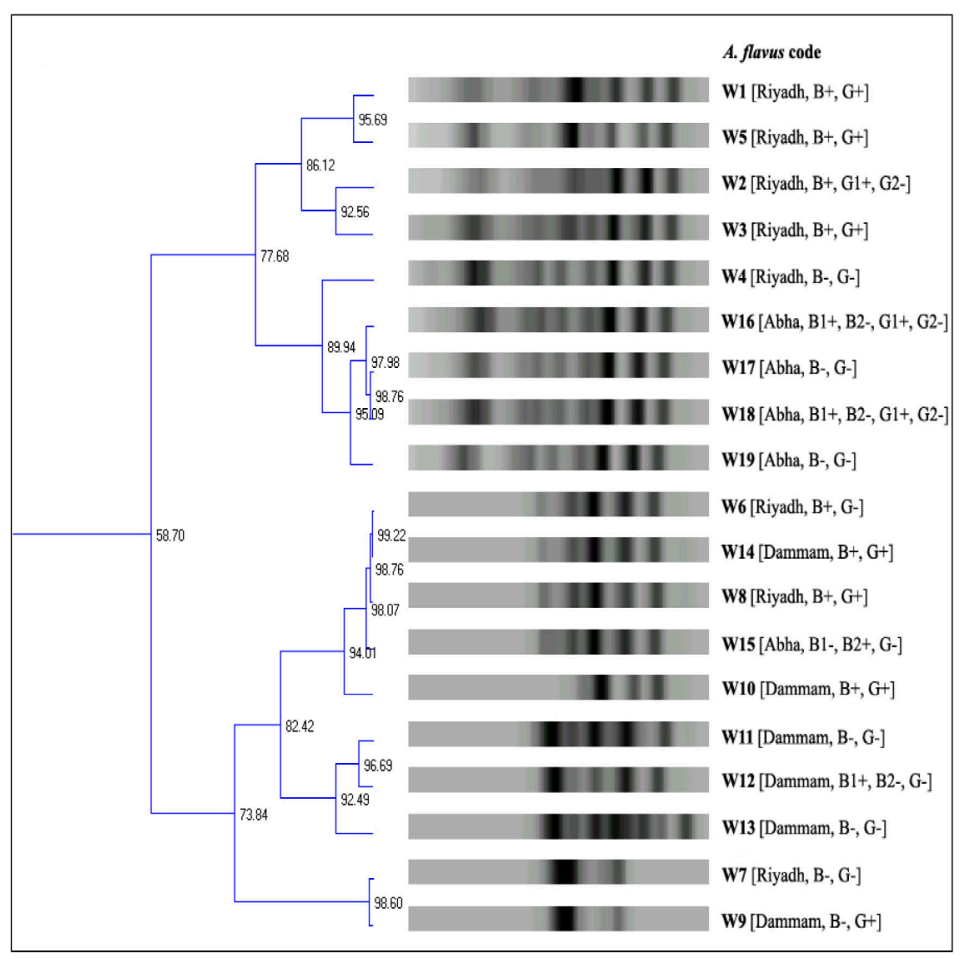

Figure 2. Dendrogram obtained by UPGMA method derived from PCR amplification banding of RAPD with RAPD primer 3 of 19 Aspergillus flavus isolates.

\section{Phenetic analysis of 19 A. flavus isolates by RAPD primer 5}

Using RAPD primer 5, cluster analysis assigned the 19 A. flavus isolates to two main clusters with similarity of $60.41-99.74 \%$ (Figure 3 ). The first main cluster included two groups with $71.27 \% \mathrm{GS}$, where the first one included two subclusters. One subcluster included Riyadh region isolates 1, 3, and 4, and Dammam region isolates 9 and 10, with GS ranging from 80.68 to $99.74 \%$. The other subcluster included Riyadh isolates 6 and 8 and Abha isolates 17 and 19 with GS ranging from 85.36 to $93.63 \%$. The second main cluster included two groups with $71.38 \%$ GS. The first one included Riyadh isolates 5 and 7 with $98.51 \%$ GS, and the second group included two subclusters with $82.68 \%$ GS. One of these subclusters included Riyadh isolate 2, Dammam isolates 13 and 14, and Abha isolates 16 and 18 with GS of 88.42$99.02 \%$. The other subcluster included Dammam isolates 11, 12, and 15, with GS ranging from 90.50 to $94.09 \%$. 


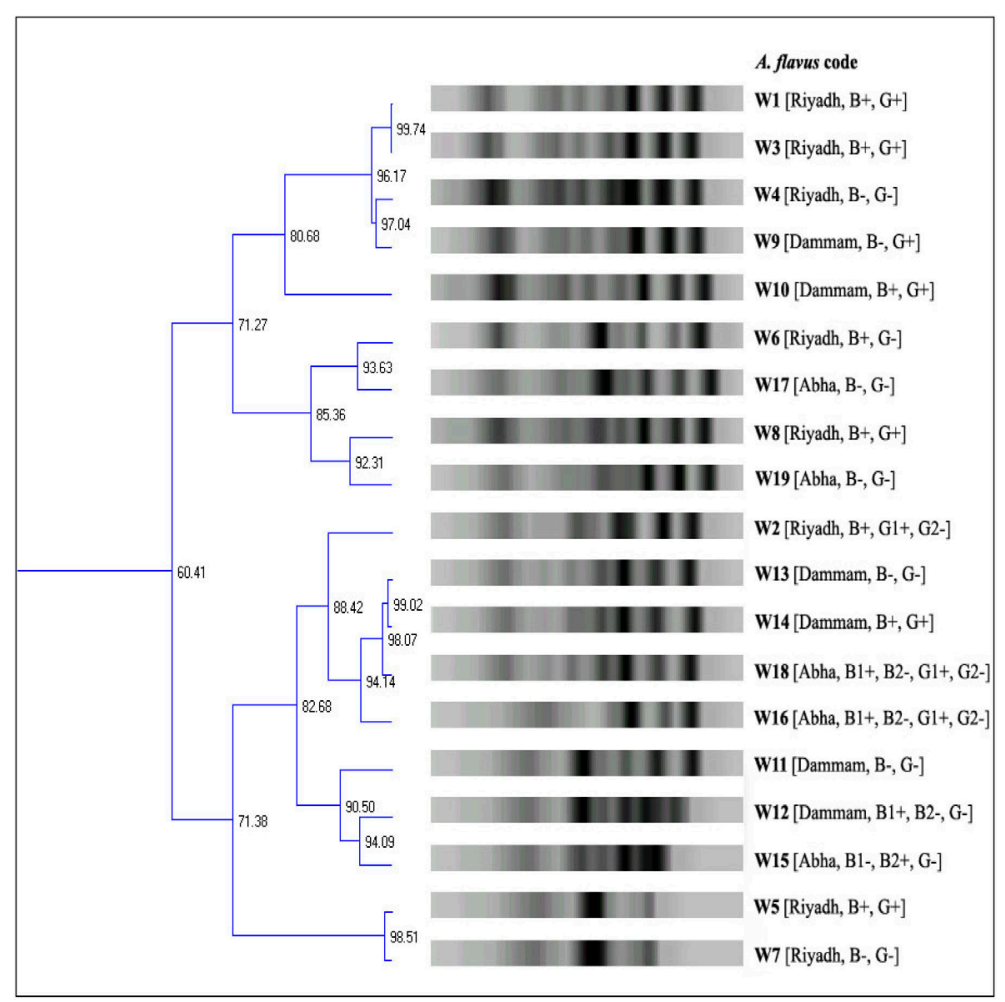

Figure 3. Dendrogram obtained by UPGMA method derived from PCR amplification banding of RAPD with RAPD primer 5 of 19 Aspergillus flavus isolates.

No correlation was found between RAPD data and geographic origin of the A. flavus isolates tested using this primer, with the exception of Dammam isolates 11, 12, and 15, which were placed in the same subcluster with $90.50 \%$ GS.

Seven subclusters included 14 isolates, 8 isolates producing AFB and AFG and 6 isolates non-producing AFB and AFG, isolated from the Riyadh, Dammam, and Abha regions; they resided in two main clusters with a random distribution. Six subclusters included a combination of 12 aflatoxigenic and non-aflatoxigenic isolates. For example, one subcluster included the two isolates W4 (AFB- and AFG-non-producing) and W9 (AFB- and AFG-producing), with 97.04\% GS. Only one subcluster included two aflatoxigenic isolates, for example, W1 and W3 with $99.74 \%$ GS. There was no clear-cut relationship between the RAPD dendrogram and AF production.

\section{Phenetic analysis using three ISSR primers}

\section{Phenetic analysis of 19 A. flavus isolates using ISSR primer $(G T G)_{5}$}

The ISSR technique was used for assessing the genetic relationships between the $19 \mathrm{~A}$. $\mathrm{fla}$ vus isolates using primer (GTG) (Figure 4). Dendrogram analysis clustered $A$. flavus isolates into two main clusters with similarity of $91.87-99.70 \%$ (Figure 4 ). The first main cluster included Ri- 
yadh region isolate 6 , showing GS of $92.71 \%$ with the remaining isolates. The second main cluster included two groups with $92.71 \%$ GS. The first one was consisted of three subclusters. These subclusters included Riyadh region isolate 3, Dammam region isolate 13, and Abha region isolates 17 and 19 , with $93.14 \%$ GS. The second group was divided into two subgroups with $93.22 \%$ GS. The first one included only Dammam region isolate 11 . The second subgroup was divided into two subclusters with $95.95 \%$ GS. The first one included only Dammam isolate 15 . The second subgroup branched into two parts with $96.67 \%$ GS. The first one included Riyadh isolates 1, 4, 5, 7, and 8 and Dammam isolates 9 and 10, ranging from 97.63 to $99.44 \%$ GS. The second part included Riyadh isolate 2, Dammam isolates 12 and 14, and Abha isolates 16 and 18, with GS ranging from 97.80 to $99.70 \%$. There was no clear-cut relationship between clustering in the ISSR dendrogram and geographic origin of the isolates tested. For example, Riyadh region isolate 7 and Dammam region isolate 9 showed high GS (99.07\%), as well as Dammam isolate 12 and Abha isolate 16 (99.70\%).

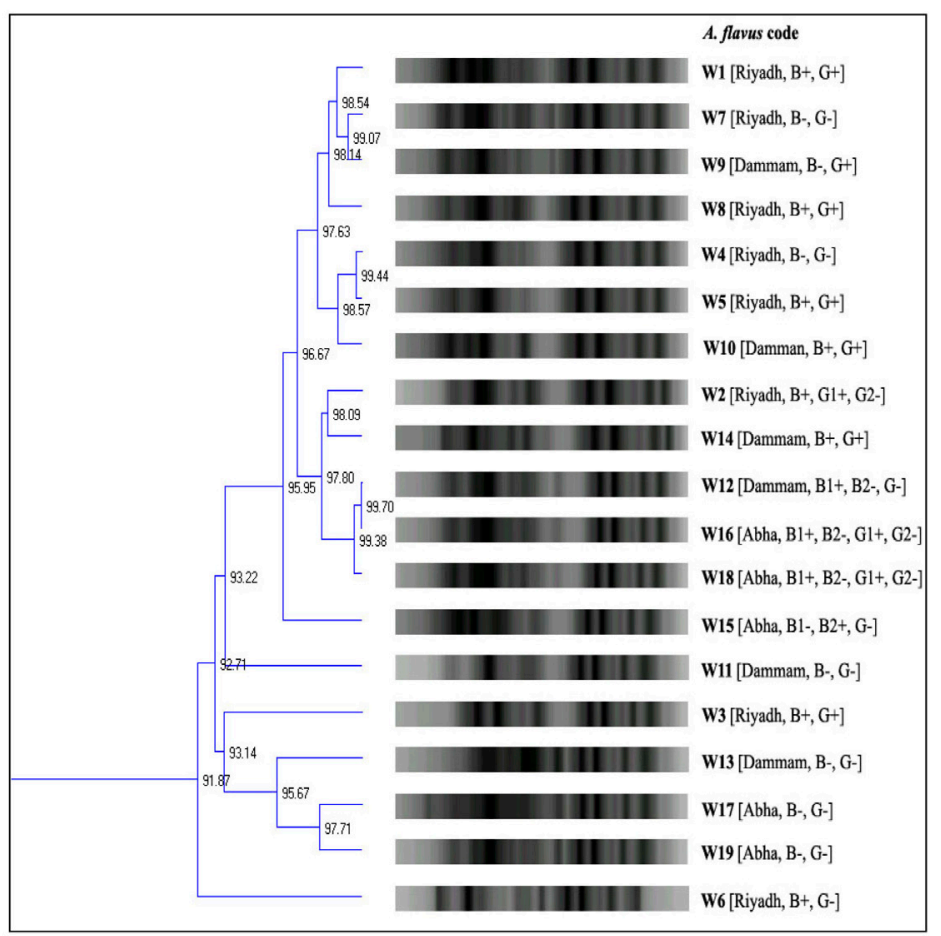

Figure 4. Dendrogram obtained by UPGMA method derived from PCR amplification banding of ISSR with primer $(\mathrm{GTG})_{5}$ of 19 Aspergillus flavus isolates.

Five subclusters included 10 isolates, 6 isolates producing AFB and AFG and 4 isolates not producing AFB and AFG, isolated from the Riyadh, Dammam, and Abha regions, and they resided in two main clusters with a random distribution. Two subclusters included a combination of four aflatoxigenic and non-aflatoxigenic isolates. For example, one subcluster included the two isolates W4 (AFB- and AFG-non-producing) and W5 (AFB- and AFG-producing) with 99.44\% GS. Two subclusters included four aflatoxigenic isolates, including W12 and W16 with 99.70\% GS. One subcluster included two non-aflatoxigenic isolates, namely W17 and W19 with $97.71 \%$ GS. 


\section{Phenetic analysis of 19 A. flavus isolates using ISSR primer $(G A C A)_{4}$}

Using the random primer (GACA), cluster analysis placed the 19 A. flavus isolates into two main clusters with GS of 91.77-99.69\% (Figure 5). The first main cluster included Riyadh region isolate 8 and Abha region isolates 16 and 19, with $93.70 \%$ GS. The second main cluster included two subgroups with $94.22 \%$ GS. The first included only Riyadh region isolate 4. The second subgroup was divided into two subclusters with $95.44 \%$ GS. The first included only Abha isolate 14. The second subcluster branched into two parts with $96.01 \%$ GS and included the remaining isolates. The first part included Riyadh isolate 7, Abha isolate 17, and Dammam isolate 11, with GS ranged $96.93-98.10 \%$. The second parts also branched into overlapping subclusters with GS ranging from 96.68 to $99.69 \%$. In these subclusters, one included Dammam isolate 12 and Abha isolate 18, with $99.35 \%$ GS, and the other one included Riyadh isolates 2, 1, 5, 3, and 6 and Dammam isolates 9, 10, and 13, with 98.30\% GS.

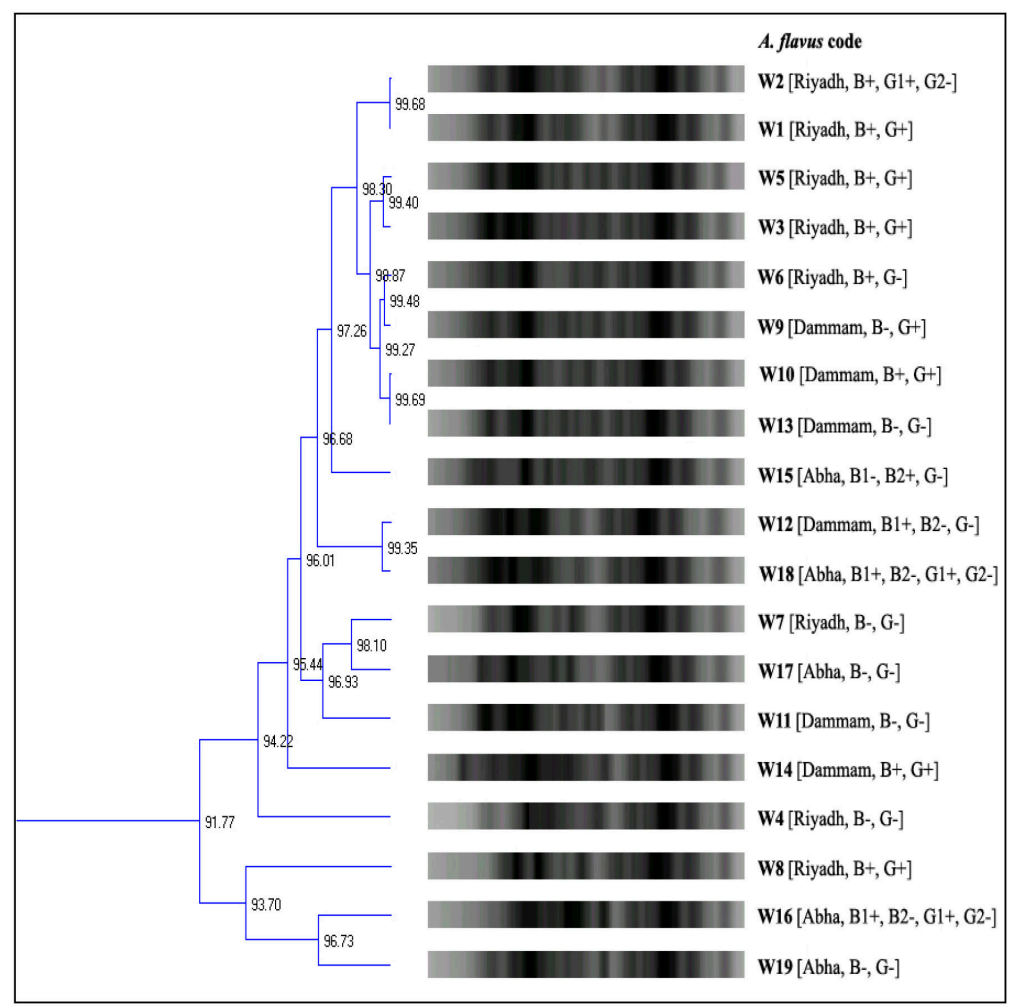

Figure 5. Dendrogram obtained by UPGMA method derived from PCR amplification banding of ISSR with primer (GACA) ${ }_{4}$ of 19 Aspergillus flavus isolates.

With reference to this primer, there was no clear-cut relationship between clustering in the ISSR dendrogram and geographic origin of the isolates tested. For example, Dammam region isolate 12 and Abha region isolate 18 showed 99.35\% GS, and 8 isolates from the Riyadh and Abha regions displayed a GS of $98.30 \%$. Six subclusters included 12 isolates, 8 
isolates producing $\mathrm{AFB}$ and $\mathrm{AFG}$ and 4 isolates not producing $\mathrm{AFB}$ and $\mathrm{AFG}$, obtained from the Riyadh, Dammam, and Abha regions, and they resided in two main clusters with a random distribution. Two subclusters included four isolates in a combination of aflatoxigenic and nonaflatoxigenic, where one subcluster included the two isolates W10 (producing AFB and AFG) and W13 (not producing AFB and AFG), with 99.69\% GS. Three subclusters included six aflatoxigenic isolates, including W5 and W3, with $99.40 \%$ GS. Only one subcluster included two non-aflatoxigenic isolates, namely W7 and W17 with $98.10 \%$ GS.

\section{Phenetic analysis of 19 A. flavus isolates using ISSR primer $(A G A G)_{4} G$}

Using the random primer (AGAG) $\mathrm{G}$, cluster analysis placed the 19 A. flavus isolates into two main clusters with GS of 91.50-99.55\% (Figure 6). The first main cluster included only Riyadh region isolate 4 . The second main cluster included two subgroups with $92.38 \%$ GS. The first one included only Abha region isolate 16. The second subgroup was divided into two subclusters with $93.45 \%$ GS. The first included only Dammam isolate 15 . The second subcluster branched into two parts with $95.92 \%$ GS. The first branched into overlapping subclusters. This part included Riyadh isolates 3 and 8, Abha isolates 17, 19, and 18, and Dammam isolates 12, 14 and 13, with GS of $96.64 \%$. The second part also branched into overlapping subclusters. This part included Riyadh isolates 1, 6, 2, 5, and 7 and Dammam isolates 11, 9, and 10 with 96.28\% GS.

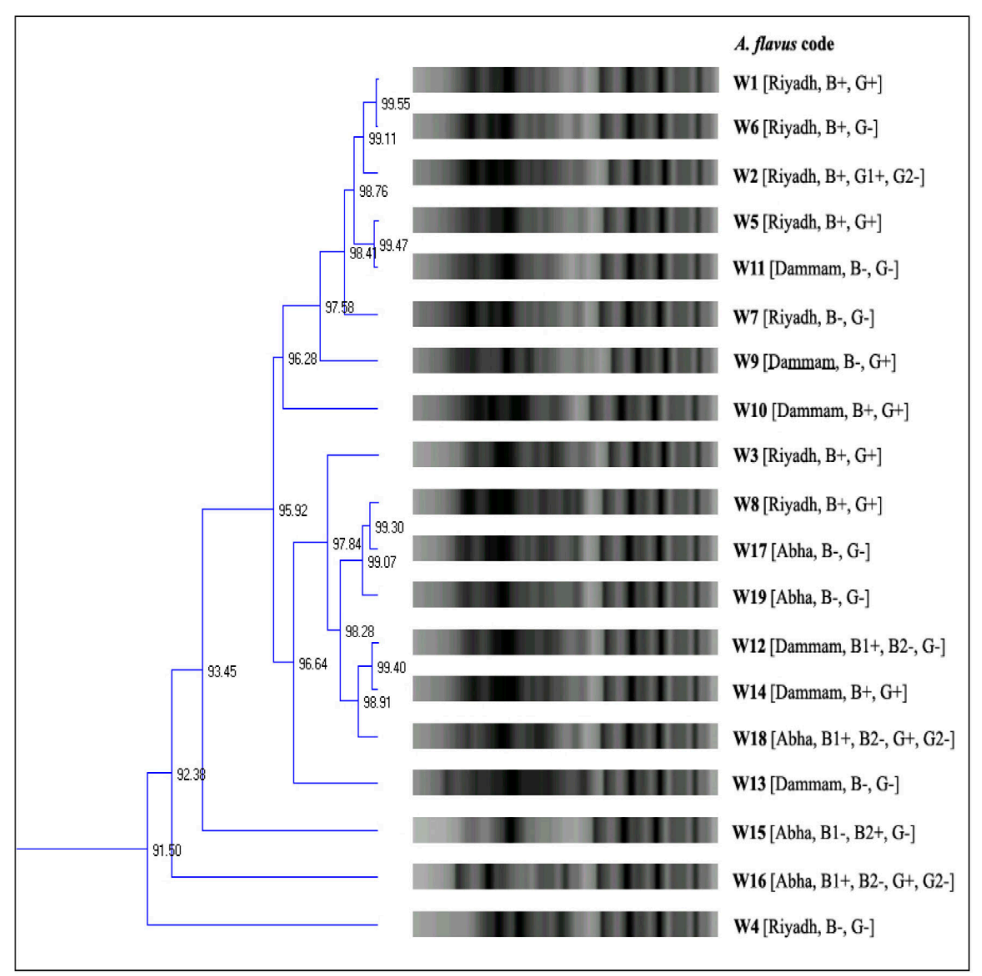

Figure 6. Dendrogram obtained by UPGMA method derived from PCR amplification banding of ISSR with primer (AGAG) ${ }_{4} \mathrm{G}$ of 19 Aspergillus flavus isolates. 
No correlation was found between ISSR data and geographic origin of the A. flavus isolates tested using this primer. For example, Riyadh region isolate 5 and Dammam region isolate 11 showed $99.47 \%$ GS, and Riyadh region isolate 8 and Abha region isolate 17 displayed $99.30 \%$ GS. Four subclusters included 8 isolates, 6 isolates producing AFB and AFG and 2 isolates not producing AFB and AFG, obtained from the Riyadh, Dammam, and Abha regions, and they resided in two main clusters with a random distribution. Two subclusters included a combination of four aflatoxigenic and non-aflatoxigenic isolates. For example, one subcluster included the two isolates W5 (producing AFB and AFG) and W11 (not producing AFB and AFG) with $99.47 \%$ GS. Two subclusters included four aflatoxigenic isolates, including W1 and W6 with $99.55 \%$ GS.

\section{DISCUSSION}

Wheat is one of the world's most important food crops. Foods made from wheat and its derivatives are a major part of a diet for over a third of the world's people. In Saudi Arabia, AFs are among the foodborne risks that are dependent upon climatic conditions. Indeed, the ability of fungi to produce mycotoxins is largely influenced by temperature, relative humidity, insect attack, storage practices, and stress conditions of the plants (Miraglia et al., 2009). A total of twelve fungi comprising six different genera (Aspergillus, Fusarium, Alternaria, Penicillium, Eupenicillium, and Rhizopus) were isolated from wheat grains in this study. The mycological profile of wheat grains includes different genera. The most common genera are Aspergillus, Penicillium, Fusarium, and Alternaria isolated from wheat grain in Saudi Arabia (Al-Hazmi, 2010; Gashgari et al., 2010). The contamination of cereal grains by fungi is a worldwide problem, where there are several reports documenting the presence of these fungi in samples from the United States (Cleveland et al., 2003), Argentina (Roige et al., 2009), Spain (Sardiñas et al., 2011), and Algeria (Riba et al., 2010).

The detection of AFs as judged by fluorescence of fungal colonies indicated that the percentage of positive samples for PDA $+\mathrm{NaCl}, \mathrm{CZ}$, and YES media were 5.8, 35.3, and $76.5 \%$, respectively. AFs were produced by some strains of $A$. flavus but not all isolates, and this has encouraged screening for fungal AF production, which commonly uses coconut agar medium for the detection of AF. In this method, aflatoxigenic strains produced a blue fluorescence on the reverse side of the colony under UV light, whereas non-aflatoxigenic ones did not produce fluorescence (Davis et al., 1987). This method was improved by adding cyclodextrin (a methylated $\beta$-cyclodextrin derivative) to common media used for testing AF production, to enhance the natural fluorescence of AFs (Fente et al., 2001).

Nineteen A. flavus isolates reported in this study were classified into eight major chemotypes of which the majority belonged to AF producers (68.4\%). Analytical methods for AFs in cereals and cereal-based products require three major steps, including extraction, clean-up, and detection/determination of the toxin (by using suitable analytical instruments/technologies). Different chromatographic methods are commonly used for quantitative determination of mycotoxins in cereals, including gas-chromatography, HPLC, and liquid chromatography-tandem mass spectrometry. HPLC affords sensitive and accurate determination of AFs in unprocessed cereals and cereal-based products (Pascale, 2009). Giray et al. (2007) analyzed 41 wheat samples in Turkey and found that $59 \%$ of the samples were contaminated with total AF; the percentage of positive samples for B1, B2, G1, and G2 were $42,12,37$, and 12\%, respectively. Seven strains 
representing $24.1 \%$ of the screened isolates produced AFs. It was found that four strains produced only B1, two strains produced B1 and B2, and one strain produced B1, G1, and G2. It was recognized that different strains of $A$. flavus vary tremendously in their ability to produce AF. These results showed that total AF occurrence in wheat flour in Jeddah (Saudi Arabia) could not be a relatively critical point, regarding quality of wheat flour (Gashgari et al., 2010). Several authors have used a selective analytical method for the determination of AFs (B1, B2, G1, and G2) and residues in cereals using HPLC for low-ppb detection.

Three primers were used to generate an RAPD dendrogram using the similarity matrix produced with the banding patterns obtained with RAPD primers 1, 3, and 5. GS for RAPD primers 1,3 , and 5 were $70.70-99.85 \%, 50.70-99.22 \%$, and $60.41-99.74 \%$, respectively, for all isolates, providing evidence of its high level of genetic diversity. High GS was revealed by RAPD and higher efficiency of the characterization of strains (Batista et al., 2008). Characterization of the seven different Aspergillus species by RAPD was useful in estimating distances between and within the same species and may help future management and conservation programs. The results indicated that genetic differences between Aspergillus species of the same genus maintain GS within this population (Irshad and Nawab, 2012).

Despite the geographic isolation, RAPD primers 1 and 3 were able to differentiate the regions somewhat. There was partial relationship between clustering in the RAPD dendrogram and geographic origin of the isolates tested. RAPD primer 3 was not able to differentiate these regions, since no correlation was found between clustering in the RAPD dendrogram and geographic origin. RAPD was used for fourteen isolates of $A$. flavus from two geographically distinct sites in Brazil. Isolates were distributed in a non-random array, but one cannot assume that this is a fully discriminatory result, and thus, RAPD usefulness is debatable (Lourenço et al., 2007). Twenty-one isolates of Rhizoctonia solani were characterized by RAPD-PCR. There was a partial relationship between GS and anastomosis groups or level of virulence or geographic origin on the basis of the RAPD dendrogram (Mahmoud et al., 2012).

With regard to RAPD markers and differentiation between aflatoxigenic and nonaflatoxigenic, RAPD primers 1, 3, and 5 showed no relationship between RAPD dendrogram and their ability to produce AF. Similar results have been described in other studies with Aspergillus spp, showing no correlation between DNA band profiles and production or non-production of AF (Lourenço et al., 2007).

The three ISSR primers were used to generate an ISSR dendrogram using the similarity matrix produced with the banding patterns obtained with primers (GTG) ${ }_{5},(\mathrm{GACA})_{4}$, and (AGAG) $)_{4}$ G. GS for these primers were $91.87-99.70 \%, 91.77-99.69 \%, 91.50-99.55 \%$, respectively, for all isolates, providing evidence of its high level of GS. This is the first study to demonstrate the use of ISSR microsatellite markers to characterize $A$. flavus isolates with wheat crop. ISSR analysis can be useful in population genetics, epidemiological surveys and ecological studies of $A$. flavus. Additionally, the $(\mathrm{GTG})_{5}$ primer can be used to generate unique products from different Aspergillus species that can be converted to a sequence-characterized amplified region to help in taxonomic identification (Batista et al., 2008). Microsatellite analysis of the Vietnamese A. flavus strains (isolated from corn and peanut) revealed a high genetic diversity, cosmopolitan and genetically connected (Tran-Dinh et al., 2009).

The microsatellite markers presented here will be very useful for investigating the diversity and population structure of A. flavus and A. parasiticus (Tran-Dinh and Carter, 2000). In view of the geographic isolation, the dendrogram produced using the ISSR marker showed 
no clear-cut relationship between clustering in the ISSR dendrogram and geographic origin. The dendrogram produced using ISSR data has shown a high GS according to geographic origin (Batista et al., 2008). A genetic relationship was shown between the 84 strains of $\mathrm{A}$. $\mathrm{fla}$ vus isolated from Vietnam. No correlation between geographic origin of strains and genotype was evident either. For example, the strains collected from both northern and southern regions were interspersed throughout the dendrogram (Tran-Dinh et al., 2009).

ISSR markers differentiated between aflatoxigenic and non-aflatoxigenic strains; ISSR primers showed a clear relationship between the ISSR dendrogram and their ability to produce AF, especially with the primers (GTG) $)_{5}$ and (GACA) $)_{4}$. A high genetic diversity has been seen in 84 strains with no evident correlation between strain toxigenicity and genotype (Tran-Dinh et al., 2009). A. flavus strains have been isolated from different oilseeds (groundnut, sunflower, and soybean), where four isolates were found to be nontoxic and eight toxic. No correlation was shown between AF production and ISSR dendrogram in a study by Hatti et al. (2010).

\section{ACKNOWLEDGMENTS}

Research supported by the King Saud University, Deanship of Scientific Research, College of Science Research Center.

\section{REFERENCES}

Al-Hazmi NA (2010). Determination of zearalenone (ZEA) in wheat samples collected from Jeddah market, Saudi Arabia. Afr. J. Microbiol. Res. 4: 2513-2519.

Amer OE, Mahmoud MA, El-Samawaty AMA and Sayed SRM (2011). Non liquid nitrogen-based-method for isolation of DNA from filamentous fungi. Afr. J. Biotechnol. 10: 14337-14341.

Bardakci F (2001). Random amplified polymorphic DNA (RAPD) markers. Turk. J. Biol. 25: 185-196.

Batista PP, Santos JF, Oliveira NT, Pires AP, et al. (2008). Genetic characterization of Brazilian strains of Aspergillus flavus using DNA markers. Genet. Mol. Res. 7: 706-717.

Bhat RV and Vasanthi S (2003). Food Safety in Food Security and Food Trade. In: Mycotoxin Food Safety Risk in Developing Countries 2020 Vision for Food, Agriculture, and the Environment, Focus 10, Brief 17. International Food Policy Research Institute, Washington, 1-12.

Castegnaro M and Wild CP (1995). IARC activities in mycotoxin research. Nat. Toxins 3: 327-331.

Cleveland TE, Dowd PF, Desjardins AE, Bhatnagar D, et al. (2003). United States Department of Agriculture-Agricultural Research Service research on pre-harvest prevention of mycotoxins and mycotoxigenic fungi in US crops. Pest Manag. Sci. 59: 629-642.

Czerwiecki L, Czajkowska D and Witkowska-Gwiazdowska A (2002). On ochratoxin A and fungal flora in Polish cereals from conventional and ecological farms. Part 2: occurrence of ochratoxin A and fungi in cereals in 1998. Food Addit. Contam. 19: 1051-1057.

Davis ND, Iyer SK and Diener UL (1987). Improved method of screening for aflatoxin with a coconut agar medium. Appl. Environ. Microbiol. 53: 1593-1595.

Domsch KH, Gams W and Anderson TH (1993). Compendium Fungi. Academic Press, London.

FAO (2009). Agribusiness Handbook 2009. Food and Ariculture Organization of United Nations, Rome.

Fente CA, Ordaz JJ, Vazquez BI, Franco CM, et al. (2001). New additive for culture media for rapid identification of aflatoxin-producing Aspergillus strains. Appl. Environ. Microbiol. 67: 4858-4862.

Franco CM, Fente CA, Vazquez BI, Cepeda A, et al. (1998). Interaction between cyclodextrins and aflatoxins Q1, M1 and P1. Fluorescence and chromatographic studies. J. Chromatogr. A 815: 21-29.

Gashgari RM, Shebany YM and Gherbawy YA (2010). Molecular characterization of mycobiota and aflatoxin contamination of retail wheat flours from Jeddah markets. Foodborne Pathog. Dis. 7: 1047-1054.

Gertz C (1990). HPLC Tips and Tricks. Great Britain at the Iden Press, Oxford.

Giray B, Girgin G, Engin AB and Aydin S (2007). Aflatoxins levels in wheat samples consumed in some regions of Turkey. Food Control 18: 23-29. 
Gonzalez HH, Resnik SL, Boca RT and Marasas WF (1995). Mycoflora of Argentinian corn harvested in the main production area in 1990. Mycopathologia 130: 29-36.

Hadrich I, Makni F, Ayadi A and Ranque S (2010). Microsatellite typing to trace Aspergillus flavus infections in a hematology unit. J. Clin. Microbiol. 48: 2396-2401.

Hadrich I, Makni F, Neji S, Cheikhrouhou F, et al. (2011). A review molecular typing methods for Aspergillus flavus isolates. Mycopathologia 172: 83-93.

Hatti AD, Taware SD, Taware AS, Pangrikar PP, et al. (2010). Genetic diversity of toxigenic and non-toxigenic Aspergillus flavus strains using ISSR markers. Int. J. Curr. Res. 5: 61-66.

IARC (1982). The Evaluation of the Carcinogenic Risk of Chemical to Humans. In: IARC Monograph Supplement, Vol. 4. International Agency for Research on Cancer, Lyon.

Irshad S and Nawab R (2012). Molecular characterization of seven different species of Aspergillus through random amplified polymorphic DNA (RAPD) and enzyme analysis. J. Microbiol. Res. 2: 47-50.

Kotsonis FN, Burdock GA and Flamm WG (2001). Food Toxicology. In: Casarett and Doull's Toxicology: The Basic Science of Poisons (Klasses CD, ed.). McGraw-Hill, New York, 1049-1088.

Lourenço A, Durigon EL, Zanotto P, Cruz Madeira JE, et al. (2007). Genetic diversity of environmental Aspergillus flavus strains in the state of Sao Paulo, Brazil by random amplified polymorphic DNA. Mem. Inst. Oswaldo Cruz 102: 687-692.

Mahmoud MA, Al-Sohaibani SA, Abdelbacki AM, Al-Othman MR, et al. (2012). Molecular characterization of the pathogenic plant fungus Rhizoctonia solani (Ceratobasidiaceae) isolated from Egypt based on protein and PCRRAPD profiles. Genet. Mol. Res. 11: 3585-3600.

Miraglia M, Marvin HJ, Kleter GA, Battilani P, et al. (2009). Climate change and food safety: an emerging issue with special focus on Europe. Food Chem. Toxicol. 47: 1009-1021.

Moss MO (1996). Mode of formation of ochratoxin A. Food Addit. Contam. 13 (Suppl): 5-9.

Neal COS, Richardson AO, Hurst SF and Tortorano AM (2011). Global population structure of Aspergillus terreus inferred by ISSR typing reveals geographical subclustering. BMC Microbiol. 16: 203-209.

Paranagama PA, Abeysekera KH, Abeywickrama K and Nugaliyadde L (2003). Fungicidal and anti-aflatoxigenic effects of the essential oil of Cymbopogon citratus (DC.) Stapf. (lemongrass) against Aspergillus flavus Link. isolated from stored rice. Lett. Appl. Microbiol. 37: 86-90.

Pascale M (2009). Detection methods for mycotoxins in cereal grains and cereal products. Proc. Nat. Sci. Matica Srpska Novi Sad 117: 15-25.

Pildain MB, Frisvad JC, Vaamonde G, Cabral D, et al. (2008). Two novel aflatoxin-producing Aspergillus species from Argentinean peanuts. Int. J. Syst. Evol. Microbiol. 58: 725-735.

Pittet A (1998). Natural occurrence of mycotoxins in food and feeds - an updated Review. Rev. Méd. Vét. 149: 479-492.

Raper KB and Fennell DI (1965). The Genus of Aspergillus. Williams \& Wilkins Company, Baltimore.

Reddy MP, Sarla N and Siddiq EA (2002). Inter simple sequence repeat (ISSR) polymorphism and its application in plant breeding. Euphytica 128: 9-17.

Reddy KRN, Reddy ChS, Nataraj Kumar P, and Reddy CS, et al. (2009). Genetic variability of aflatoxin B $_{1}$ producing Aspergillus flavus strains isolated from discolored rice grains. World J. Microbiol. Biotechnol. 25: 33-39.

Riba A, Bouras N, Mokrane S, Mathieu F, et al. (2010). Aspergillus section Flavi and aflatoxins in Algerian wheat and derived products. Food Chem. Toxicol. 48: 2772-2777.

Roige MB, Aranguren SM, Riccio MB, Pereyra S, et al. (2009). Mycobiota and mycotoxins in fermented feed, wheat grains and corn grains in Southeastern Buenos Aires Province, Argentina. Rev. Iberoam. Micol. 26: 233-237.

Sardiñas N, Vázquez C, Gil-Serna J, González-Jaén MT, et al. (2011). Specific detection and quantification of Aspergillus flavus and Aspergillus parasiticus in wheat flour by SYBR(R) Green quantitative PCR. Int. J. Food Microbiol. 145: 121-125.

Tran-Dinh N and Carter D (2000). Characterization of microsatellite loci in the aflatoxigenic fungi Aspergillus flavus and Aspergillus parasiticus. Mol. Ecol. 9: 2170-2172.

Tran-Dinh N, Kennedy I, Bui T and Carter D (2009). Survey of Vietnamese peanuts, corn and soil for the presence of Aspergillus flavus and Aspergillus parasiticus. Mycopathologia 168: 257-268.

Wiese MV (1987). Compendium of Wheat Diseases. 2nd edn. APS Press, St. Paul. 